\title{
Effect of different surface treatment on the repair bond strength of feldspathic porcelain
}

\author{
Azam Valian ${ }^{1, A}$, Elham Moravej Salehi' ${ }^{2, E}$, Mehrangar Mahmoudzadeh ${ }^{3, F}$, Neda Kheirkhah Dabagh ${ }^{1, B-D}$ \\ ${ }^{1}$ Department of Restorative Dentistry, Shahid Beheshti University of Medical Sciences, Tehran, Iran \\ 2 Private dental practice, Tehran, Iran \\ ${ }^{3}$ Department of Periodontics, Isfahan University of Medical Sciences, Iran \\ A - research concept and design; B - collection and/or assembly of data; $\mathrm{C}$ - data analysis and interpretation; \\ $D$ - writing the article; $\mathrm{E}$ - critical revision of the article; $\mathrm{F}$ - final approval of the article
}

\section{Address for correspondence \\ Neda Kheirkhah Dabagh \\ E-mail: kheirkhahneda.nk@gmail.com}

\section{Funding sources}

The study was financially supported by the Research Institute of Shahid Beheshti University of Medical Sciences, Tehran, Iran.

Conflict of interest

None declared

Received on November 29, 2019

Reviewed on January 26, 2020

Accepted on November 4, 2020

Published online on March 31, 2021

Cite as

Valian A, Salehi EM, Mahmoudzadeh M, Dabagh NK. Effect of different surface treatment on the repair bond strength of feldspathic porcelain. Dent Med Probl. 2021;58(1):107-113. doi:10.17219/dmp/130101

DOI

$10.17219 / \mathrm{dmp} / 130101$

Copyright

○) 2021 by Wroclaw Medical University

This is an article distributed under the terms of the

Creative Commons Attribution 3.0 Unported License (CC BY 3.0)

(https://creativecommons.org/licenses/by/3.0/).

\begin{abstract}
Background. Considering the use of silane-containing universal adhesives to enhance the repair bond strength of porcelain restorations, a question arises whether the application of these adhesives eliminates the need for a separate application of silane or not.

Objectives. This study aimed to assess the effect of various kinds of surface treatment, including hydrofluoric acid (HF) etching, the application of bis-silane and the use of universal adhesives, on the repair bond strength of feldspathic porcelain.

Material and methods. In this experimental in vitro study, 70 porcelain disks were fabricated and divided into 7 groups $(n=10)$ for the following types of surface treatment: ( (control group) - HF etching + silane + Porcelain Bond; HSB - HF etching + Single Bond; HSSB - HF etching + silane + Single Bond; $\mathrm{HAB}-\mathrm{HF}$ etching + All Bond; HSAB - HF etching + silane + All Bond; HFB - HF etching + FuturaBond ${ }^{\circledR}$; and HSFB - HF etching + silane + FuturaBond. After applying different kinds of surface treatment, the specimens were light-cured and the Filtek ${ }^{\circledR} Z 250$ composite was bonded to the treated surfaces. The specimens were incubated in distilled water at $37^{\circ} \mathrm{C}$ for $24 \mathrm{~h}$, and then underwent 5,000 thermal cycles. The repair bond strength of porcelain was measured and the mode of failure was determined under a stereomicroscope. Data was analyzed using the one-way analysis of variance (ANOVA) and Tukey's test.
\end{abstract}

Results. Differences between the groups in the porcelain repair bond strength were significant $(p<0.0001)$. Bond strength for Single Bond $(p<0.001)$ and All Bond $(p<0.001)$ along with silane was significantly higher than for the application of these adhesives without a separate silane application step. This difference was not significant for FuturaBond. Mixed failure was the dominant mode of failure in all groups.

Conclusions. The application of silane, irrespective of the use of universal adhesives with or without silane, increased the porcelain repair bond strength. Thus, a separate silane application step following HF etching and the use of universal adhesives with or without silane can enhance the repair bond strength of feldspathic porcelain.

Key words: dental adhesives, shear strength, dental porcelain, silanes, dental etching, hydrofluoric acid 


\section{Introduction}

Glass-ceramics and feldspathic porcelains are 2 premier esthetic materials widely utilized to prepare full-ceramic or metal-ceramic crowns for custom veneer restorations in esthetic dentistry. These materials provide a biocompatible and corrosion-resistant veneering layer as well as an esthetic appearance. ${ }^{1-3}$ Due to their excellent esthetic features, they are suitable for ceramic laminate veneers, inlays and onlays. ${ }^{4}$ However, porcelain restorations may fail in the oral environment due to occlusal forces, trauma, internal defects, or an inappropriate design. ${ }^{3,5}$ Clinical studies have also reported the prevalence of fracture of ceramic restorations to be $5-10 \%$, following 10 years of clinical service in the oral cavity. ${ }^{6}$ Due to such factors as a high cost of fabrication of a new restoration, saving time and difficult retrieval of old restorations, the repair or reconstruction of ceramic or metal-based restorations has been suggested as a clinical solution. ${ }^{5,7}$

Restorative procedures include indirect methods (laboratory repairing techniques) and/or direct techniques, which use resin composite materials intraorally for repairing the fractured porcelain. ${ }^{1,7,8}$ Current direct restoration methods involve micromechanical and chemical bonding as well as some treatment for preparing the ceramic surface, which affects the resin-porcelain bonding. ${ }^{3}$ Ceramic surfaces can be prepared by means of several methods, including acid etching with hydrofluoric acid (HF), ${ }^{9,10}$ air abrasion with $\mathrm{AL}_{2} \mathrm{O}_{3}$ particles, ${ }^{9,11}$ silica coating, ${ }^{3}$ and laser irradiation. ${ }^{12,13}$ These procedures are applied to increase surface roughness and improve bond strength. ${ }^{3,14}$ Silane coupling components are utilized not only to improve the wettability of the ceramic surface, but also to chemically strengthen the ceramic-composite resin bond. 3,9 Silane bilaterally attaches to silicon dioxide groups and methacrylate groups, bonding the ceramic surface to the resin. ${ }^{9,15}$ Therefore, silane makes the ceramic surface both organophilic and hydrophobic, which plays a pivotal role in establishing the ceramicresin chemical bond. ${ }^{3}$

Recently, a new generation of bonding agents, known as universal adhesives, has been introduced. They provide a durable, long-term bond to different substrates, such as zirconia, silica-based ceramics, non-silica-based ceramics, noble and non-noble metals, and stainless steel. ${ }^{16}$

\section{Objectives}

This study aimed to assess the effect of different kinds of surface treatment, including HF etching, the application of a two-part silane coupling agent and the use of universal adhesives, on the repair bond strength of feldspathic porcelain in vitro. The null hypothesis was that different surface treatment methods do not affect the strength of the repair bond of the composite resin to ceramic blocks.

\section{Material and methods}

This experimental in vitro study evaluated 70 feldspathic porcelain disks measuring $6 \mathrm{~mm} \times 2 \mathrm{~mm}\left(\right.$ Ceramco ${ }^{\circledR} 3$; Dentsply, York, USA). The specimens were inspected under a magnifier at $\times 10$ magnification to ensure the absence of microcracks. For the standardization of porcelain surfaces, they were polished with 400- and 600-grit silicon carbide disks under running water for $15 \mathrm{~s}$. The specimens were rinsed with water and dried. After that, they were cleaned with phosphoric acid, and then 9.5\% HF (Porcelain Etchant gel; Bisco, Schaumburg, USA) was applied on the surfaces of the specimens for $2 \mathrm{~min}$. The specimens were then rinsed with water for $1 \mathrm{~min}$ and dried with air spray for $1 \mathrm{~min}$. Next, they were subjected to the following interventions:

- group C (control): One layer of a two-part silane coupling agent (Bis-Silane Parts A \& B; Bisco) was applied on the surfaces of the specimens with a microbrush for $1 \mathrm{~min}$ and dried with air spray for $30 \mathrm{~s}$. Next, 1 layer of porcelain bonding resin (Porcelain Bond; Bisco) was applied on the surfaces of the specimens and light-cured for $20 \mathrm{~s}$ with a light-curing unit (Demetron ${ }^{\circledR}$ Optilux 401 device; Kerr, Danbury, USA) at a light intensity of $600 \mathrm{~mW} / \mathrm{cm}^{2}$; - group HSB: One layer of the silane-containing Single Bond Universal adhesive (3M ESPE, St. Paul, USA) was applied on the surfaces of the specimens in this group. The bonding agent was gently rubbed on the surface with an applicator for $15 \mathrm{~s}$ and thinned with gentle air spray for $5 \mathrm{~s}$ to evaporate the solvent. The specimens were light-cured for $20 \mathrm{~s}$ as described for group C;

- group HSSB: One layer of bis-silane (Bis-Silane Parts A \& B; Bisco) was applied on the surfaces of the specimens with a microbrush for $1 \mathrm{~min}$. The silanized surfaces were then dried with air spray for $30 \mathrm{~s}$. Next, 1 layer of the silane-containing Single Bond Universal adhesive (3M ESPE) was applied on the surfaces as explained for group HSB and light-cured for $20 \mathrm{~s}$ as described for the previous groups;

- group HAB: One layer of the All Bond Universal bonding agent (Bisco) was applied on the surfaces of the specimens and light-cured for $20 \mathrm{~s}$ as described for the previous groups;

- group HSAB: One layer of bis-silane (Bis-Silane Parts A \& B; Bisco) along with 1 layer of All Bond Universal (Bisco) were applied on the surfaces of the specimens and light-cured for $20 \mathrm{~s}$ as described for the previous groups;

- group HFB: One layer of the FuturaBond ${ }^{\circledR}$ Universal bonding agent (Voco, Cuxhaven, Germany) was applied on the surfaces of the specimens and light-cured for $20 \mathrm{~s}$ as described for the previous groups;

- group HSFB: One layer of bis-silane (Bis-Silane Parts A \& B; Bisco) along with 1 layer of FuturaBond Universal (Voco) were applied on the surfaces of the specimens and light-cured for $20 \mathrm{~s}$ as described for the previous groups. 
The A1 shade of the Filtek ${ }^{\circledR}$ Z250 nanofilled composite (3M ESPE) at a thickness of $2 \mathrm{~mm}$ was applied into transparent, cylindrical Tygon ${ }^{\circledR}$ tubes measuring $3 \mathrm{~mm} \times 4 \mathrm{~mm}$. The tubes were placed on the bonding surface. Each increment was light-cured for $40 \mathrm{~s}$. Composite cylinders were additionally cured for $120 \mathrm{~s}$ from the porcelain surface in all 3 dimensions at an angle of $45^{\circ}$. Next, the Tygon tubes were separated and the specimens were inspected under a stereomicroscope (Nikon, Tokyo, Japan) at $\times 10$ magnification. Then, the specimens were immersed in distilled water and placed in an incubator (Pars Azma Co., Tehran, Iran) at $37^{\circ} \mathrm{C}$ for $24 \mathrm{~h}$. Next, the specimens underwent 5,000 thermal cycles at $5-55^{\circ} \mathrm{C}$ with a dwell time of $30 \mathrm{~s}$ and a transfer time of $10 \mathrm{~s}$ in a thermocycler (TC-300; Vafaei Industrial, Tehran, Iran). The specimens were then mounted in auto-polymerizing acrylic resin (Acropars; Marlic Co., Tehran, Iran) and transferred to a universal testing machine (Z050; ZwickRoell, Ulm, Germany) to measure shear bond strength (SBS). A load was applied to the composite-porcelain interface at a crosshead speed of $1 \mathrm{~mm} / \mathrm{min}$ until debonding occurred. The load at debonding was recorded and bond strength was calculated in megapascals (MPa).

The composition of the materials used in the study is presented in Table 1.

The mode of failure of the specimens was determined under a stereomicroscope (Nikon) at $\times 20$ magnification. The mode of failure was categorized as adhesive, cohesive or mixed (adhesive/cohesive). One specimen from each category of failure was inspected under a scanning electron microscope (Nikon) at $\times 10$, $\times 50$ and $\times 100$ magnification to further analyze the failure mode in detail.

\section{Statistical analysis}

Data was analyzed using IBM SPSS Statistics for Windows, v. 21.0 (IBM Corp., Armonk, USA). The mean $(M)$, standard deviation $(S D)$, standard error $(S E)$, and upper and lower bounds with $95 \%$ confidence intervals (CIs) of the repair bond strength of feldspathic porcelain in different surface treatment groups were calculated and reported. First, the normal distribution of data was evaluated using the KolmogorovSmirnov test. Since data was normally distributed, the effect of the application of bis-silane and a universal adhesive on the repair bond strength of porcelain was separately analyzed using the two-way analysis of variance (ANOVA). The repair SBS of feldspathic porcelain in different groups was compared using one-way ANOVA. Since the result of one-way ANOVA was significant, pairwise comparisons were performed using Tukey's multiple comparison test. The level of significance was set at $0.05(\alpha=0.05)$.

\section{Results}

Table 2 presents the mean values of the repair SBS of feldspathic porcelain in different surface treatment groups.

According to the results of one-way ANOVA, significant differences were found in the repair bond strength of feldspathic porcelain between different surface treatment groups $(p<0.001)$. In other words, the application of HF, universal adhesives and silane had significantly different effects on the repair bond strength of feldspathic porcelain.

Table 1. Characteristics of the selected studies

\begin{tabular}{|c|c|c|}
\hline Material & Manufacturer & Components \\
\hline Porcelain Etchant & $\begin{array}{l}\text { Bisco, } \\
\text { Shaumburg, USA }\end{array}$ & $9.5 \% \mathrm{HA}$ \\
\hline Bis-silane & $\begin{array}{l}\text { Bisco, } \\
\text { Shaumburg, USA }\end{array}$ & ethanol, silane coupling agent, water \\
\hline Porcelain bonding resin & $\begin{array}{l}\text { Bisco, } \\
\text { Shaumburg, USA }\end{array}$ & Bis-GMA, TEGDMA, UDMA \\
\hline Single Bond Universal & $\begin{array}{l}\text { 3M ESPE, } \\
\text { St. Paul, USA }\end{array}$ & $\begin{array}{l}\text { HEMA, dimethacrylate, MDP monomer, silane methacrylate-modified polyalkenoic acid copolymer, } \\
\text { ethanol, water, initiators, Vitrebond }{ }^{\mathrm{TM}} \text { copolymer }\end{array}$ \\
\hline All Bond Universal & $\begin{array}{l}\text { Bisco, } \\
\text { Shaumburg, USA }\end{array}$ & 10-MDP dimethacrylate resin, HEMA, ethanol, water, initiators \\
\hline FuturaBond ${ }^{\circledR}$ Universal & $\begin{array}{l}\text { Voco, } \\
\text { Cuxhaven, Germany }\end{array}$ & modified 10-MDP dimethacrylate resin, HEMA, ethanol, water, carboxylic acid ester, initiators \\
\hline $\begin{array}{l}\text { Filtek }^{\circledR} \mathrm{Z} 250 \\
\text { composite resin }\end{array}$ & $\begin{array}{l}\text { 3M ESPE, } \\
\text { St. Paul, USA }\end{array}$ & $\begin{array}{l}\text { Bis-GMA, Bis-EMA, TEGDMA, zirconia/silica fillers } \\
\text { filler volume: } 60 \% \\
\text { filler size: } 0.01-3.5 \mu\end{array}$ \\
\hline
\end{tabular}

HA - hydrofluoric acid; Bis-GMA - bisphenol A-glycidyl methacrylate; TEGDMA - triethylene glycol dimethacrylate; UDMA - urethane dimethacrylate; HEMA - hydroxyethyl methacrylate; MDP - methacryloyloxydecyl dihydrogen phosphate; Bis-EMA - ethoxylated bisphenol A-glycidyl methacrylate. 
Table 2. Repair shear bond strength (SBS) [MPa] of feldspathic porcelain for surface treatment with universal adhesives, silane and hydrofluoric acid (HF)

\begin{tabular}{|l|cc|c|}
\multicolumn{1}{c|}{ Group } & $\begin{array}{c}\text { SBS } \\
{[\mathrm{MPa}]}\end{array}$ & Min & Max \\
\hline $\mathrm{C}^{\mathrm{a}}$ & $13.31 \pm 1.16$ & 11.22 & 15.23 \\
HSB $^{\mathrm{b}}$ & $11.39 \pm 1.21$ & 9.80 & 13.70 \\
HSSB $^{\mathrm{a}}$ & $14.08 \pm 1.39$ & 11.27 & 16.11 \\
HAB $^{\mathrm{b}}$ & $11.21 \pm 0.71$ & 10.10 & 12.26 \\
HSAB $^{\mathrm{a}}$ & $13.92 \pm 1.62$ & 11.27 & 16.80 \\
HFBa, $^{\mathrm{a} b}$ & $12.27 \pm 1.59$ & 9.80 & 14.80 \\
HSFB $^{\mathrm{a}}$ & $13.66 \pm 1.61$ & 11.37 & 16.66 \\
\hline
\end{tabular}

Groups: $\mathrm{C}$ (control group) - HF + bis-silane + Porcelain Bond;

$\mathrm{HSB}-\mathrm{HF}+$ Single Bond; $\mathrm{HSSB}-\mathrm{HF}+$ bis-silane + Single Bond;

$\mathrm{HAB}-\mathrm{HF}+$ All Bond; $\mathrm{HSAB}-\mathrm{HF}+$ bis-silane + All Bond;

$\mathrm{HFB}-\mathrm{HF}+$ FuturaBond $^{\circledR} ; \mathrm{HSFB}-\mathrm{HF}+$ bis-silane + FuturaBond ${ }^{\circledR}$;

min - minimum; max - maximum; different superscript letters indicate

statistically significant differences between various surface treatment

methods $(p<0.05)$

Data presented as mean $(M) \pm$ standard deviation (SD)

Two-way ANOVA revealed that the effect of an additional silane application step on the repair bond strength of porcelain was significant $(p<0.0001)$. However, the effect of the type of universal adhesive $(p=0.660)$ as well as the interaction effect of the type of universal adhesive and an additional bis-silane application step $(p=0.240)$ on the repair bond strength of feldspathic porcelain were not significant. In other words, the application of bis-silane increased the repair bond strength irrespective of the type of universal adhesive, with or without silane in its composition.

The current findings revealed significant differences in the repair bond strength of feldspathic porcelain following the application of bis-silane and universal adhesives with or without silane. The maximum repair bond strength of porcelain was noted following the application of $\mathrm{HF}$ along with bis-silane and Single Bond containing silane $(14.08 \mathrm{MPa})$. The minimum bond strength was noted following HF etching along with the application of the All Bond adhesive without silane, without the additional application of bis-silane (11.21 MPa).

Pairwise comparisons revealed that the bond strength following the application of $\mathrm{HF}+$ bis-silane + Single Bond containing silane was significantly higher than the bond strength following the application of $\mathrm{HF}+$ Single Bond containing silane without a separate application of bissilane $(p<0.001)$. On the other hand, the bond strength following the application of $\mathrm{HF}+$ bis-silane + All Bond was significantly higher than that following the application of HF + All Bond without silane $(p<0.001)$. The porcelain repair bond strength following the application of HF + bis-silane + FuturaBond was higher than that following the application of $\mathrm{HF}+$ FuturaBond without silane; however, this difference did not reach statistical significance $(p>0.05)$. In all groups, the application of bis-silane increased the porcelain repair bond strength irrespective of the presence or absence of silane in the composition of the universal adhesive used. However, in the case of FuturaBond, which does not contain silane, the application of bis-silane could not significantly increase bond strength.

Table 3 shows different modes of failure in different surface treatment groups. No case of adhesive failure was noted in any group, and mixed failure was the dominant mode of failure in all groups. Cohesive failure was noted only in a few specimens.

Table 3. Modes of failure in different surface treatment groups $(n=10)$

\begin{tabular}{|l|c|c|c|c|}
\multirow{2}{*}{ Group } & \multicolumn{5}{|c|}{ Mode of failure [\%] } \\
\cline { 2 - 5 } C & cohesive & mixed & adhesive & total \\
\hline HSB & 20 & 80 & - & 100 \\
HSSB & - & 100 & - & 100 \\
HAB & 20 & 80 & - & 100 \\
HSAB & - & 100 & - & 100 \\
HFB & 30 & 70 & - & 100 \\
HSFB & 10 & 100 & - & 100 \\
Total & 11.4 & 80 & - & 100 \\
\hline
\end{tabular}

\section{Discussion}

This study evaluated the effect of different kinds of surface treatment, including $\mathrm{HF}$ etching, bis-silane and universal adhesives, on the repair bond strength of feldspathic porcelain in vitro. Considering the results, the null hypothesis that different surface treatment methods do not affect the repair bond strength of the composite to ceramic blocks was rejected.

Adhesive dentistry have recently enabled the use of adhesives for the repair of ceramic restorations instead of their replacement. ${ }^{16}$ In the present study, Porcelain Bond along with bis-silane and HF conditioning was used for surface treatment in the control group. The porcelain repair bond strength values in the experimental groups were compared with the value in the control group. This surface treatment modality is commonly used for conditioning the porcelain surface prior to porcelain repair. ${ }^{14}$ On the other hand, Filtek Z250 was used to enable the comparison of the results. Accordingly, the HF etching and silanization methods actually improved the repair bond strength of feldspathic porcelain. ${ }^{15}$ Etching porcelain with HF selectively dissolves the glassy phase, resulting in irregular microporosity and an increased surface area, and creating a micromechanical interlock, which enhances the penetration of restorative materials into tiny undercuts in the etched porcelain surface and reinforces 
the formation of hydroxyl groups on the porcelain surface. ${ }^{9,17}$ The etching of the ceramic surface results in the formation of volatile silicon tetrafluoride, which creates a soluble ionic complex (hexafluorosilicate) with HF. The molecules in the chemical composition of the etched surface can bond to proton to form tetrafluorosilicic acid. ${ }^{18}$ Dental clinicians should be well aware of the serious risk of exposure of oral tissues to HF, especially in cases when rubber dam isolation cannot be performed.

However, the gel-etching of glass-ceramics is still considered the golden standard method. This conditioning medium is considered advantageous as compared to the airabrasion method, which leaves microcracks in the ceramic. ${ }^{10}$

After applying the gel, the organic hydrolyzed functional groups react with the surface hydroxyl groups present in the inorganic substrate and create a siloxane bond $(\mathrm{Si}-\mathrm{O}-\mathrm{Si})$, producing a water molecule by-product. The organic non-hydrolyzed functional groups have carboncarbon double bonds and can be polymerized with composite resin monomers, which contain double bonds., ${ }^{9,18}$

It seems that there exists an equilibrium between the number of the exposed hydroxyl groups present in the inorganic substrate and the hydrolyzed functional groups present in silane; the quality of the siloxane bond can be predicted based on the concentration of the silane solution..$^{18}$

The surface treatment protocol also plays a role in this regard by quantifying the number of the exposed hydroxyl groups.

Silane can bond to silica fillers as well as react with organic materials, such as composite resin, and inorganic materials, such as porcelain, via its functional groups and dual reactions, and enhance their surface wettability as such. ${ }^{15}$ The present study indicated that a separate bissilane application step increased the repair bond strength of feldspathic porcelain in all groups.

To reduce the clinical stages of the adhesive procedures, manufacturers have incorporated a silane coupling agent in the composition of some universal adhesives, claiming that there is no extra need for a separate ceramic primer for chemical bonding to a glass-ceramic ${ }^{19}$ (among the materials used in the present study, Single Bond contains silane in its composition, while All Bond and FuturaBond are devoid of it). The results of the present study showed that the additional step of bis-silane application along with the use of universal adhesives with or without silane in their composition significantly increased the repair bond strength of feldspathic porcelain. Although in the case of using universal adhesives with or without silane the repair bond strength of porcelain was statistically lower than that following the use of universal adhesives along with a separate application of bis-silane, this difference did not seem to be clinically significant. However, considering the significance of high repair bond strength of porcelain, the additional step of silane application should not be eliminated so that the maximum bond strength could be achieved.
Our results are in agreement with those reported by Zaghloul et al., ${ }^{20} \mathrm{Kim}$ et al., ${ }^{21}$ Kalavacharla et al., ${ }^{22}$ Vasconcelos Moro et al., ${ }^{23}$ AlRabiah et al., ${ }^{24}$ SuárezMoya et al., ${ }^{14}$ and Yao et al., ${ }^{25}$ who claimed that a separate application of silane along with HF etching and the application of a universal adhesive significantly increased the repair bond strength of feldspathic porcelain.

Universal adhesives show decreased performance when they are used as ceramic primers, which is suggested, as the silane coupling agent is less stable in the water acidic adhesive solution. The silanol groups $(-\mathrm{Si}-\mathrm{OH})$ produced from hydrolyzing the silane groups $\left(-\mathrm{Si}-\mathrm{CH}_{3}\right)$ of the silane coupling agent in the presence of water can adsorb and chemically bond to the glass. ${ }^{15,19}$ Still, if silanol groups undergo dehydroxylation after hydrolysis, they condense and form a siloxane $(-\mathrm{O}-\mathrm{Si}-\mathrm{O})_{\mathrm{n}}$ oligomer, which does not adsorb to glass. The coupling capacity of the silane content to silica can be affected by any interaction between the different monomers of the universal adhesive, such as the presence of bisphenol A-glycidyl methacrylate (BisGMA), which prevents the silanol group and the substrate from the condensation reaction. ${ }^{19}$

Furthermore, some functional components of universal adhesives other than methacryloyloxydecyl dihydrogen phosphate (MDP), such as dimethacrylates and other additives, are reported to affect bond strength. ${ }^{19}$ These adhesive components improve the mechanical and bonding properties of the polymer by making it hydrophobic and reducing the ceramic-resin contact angle. This reduction leads to a closer interaction, which strengthens the bond. ${ }^{19}$

Functional monomers, cross-linking monomers, solvents, inhibitors, and activators may be present in the composition of universal adhesives in variable amounts and may affect bond strength as well. Undoubtedly, the amounts of monomers, diluting agents and the filler volume of different products are different, depending on their manufacturing technology. Also, limited information is available regarding the shrinkage and strength of these adhesives, which can affect their physical and mechanical properties. Other influential factors in this respect include the application technique, differences between operators and inadequate time for drying the primer, which can affect bond strength following the application of universal adhesives on different substrates.

One surface pre-treatment method recognized as effective in enhancing the bond strength of surface micromechanical interlocking junctions and improving their adhesiveness is the erbium-doped yttrium aluminum garnet (Er:YAG) laser irradiation. ${ }^{26}$ However, the controversial results of the Er:YAG laser application in ceramic surface pre-treatment, such as those reported by Akyl et al. ${ }^{27}$ and Shirinzad et al., ${ }^{28}$ where the Er:YAG laser provided a lower bond strength, different power settings and ceramic materials should be considered for the clinical use of the Er:YAG laser irradiation. For example, while high power 
settings may cause crystal or matric destruction and create a heat-damaged layer, low power settings have a poor impact on the ceramic surface. ${ }^{13}$ Most researches deduce that a water layer is created on the ceramic surface with a water-spraying device, causing the destruction of the local crystals or matrix of the ceramic due to a high temperature or pressure during laser irradiation. Accordingly, the dispersed pits formed on the ceramic surface alter the mechanical retention and affect the bond strength of the repaired ceramic. ${ }^{13}$ Therefore, setting the most suitable power is essential for the practical use of the Er:YAG laser.

However, more studies are required to establish the efficiency of the Er:YAG laser, as, for example, a study by Hou et al. showed that the Er:YAG laser could not be applied for all types of computer-aided design/computeraided manufacturing (CAD/CAM) ceramics. ${ }^{13}$ On the other hand, there are confirmative studies that suggest complementary treatment to be beneficial for increasing the efficiency of laser irradiation. A considerable instance is a report by Ahrari et al., ${ }^{12}$ suggesting the use of HF after laser irradiation in order to provide more efficient bond strength between the resin composite and feldspathic porcelain.

The current results showed that mixed failure was the dominant mode of failure in all groups and no case of adhesive failure was noted in any group. Cohesive failure ranked second after mixed failure, but with a much lower frequency; however, both were usually correlated with high values of SBS. The mode of failure is a criterion to determine the success of adhesive restorations. In this respect, adhesive failure is unacceptable, while mixed failure is acceptable and cohesive failure indicates ideal restorations. ${ }^{29}$ In a study by de Melo et al., porcelain with a lesser crystalline phase and a greater glassy phase was shown to cause more cohesive failure. ${ }^{30}$

In the present study, the specimens underwent thermocycling before SBS assessment in order to test the durability of adhesives and simulate intraoral aging. Thermal cycles cause hydrolytic and thermal reductions, and by sudden thermal alterations, they simulate thermal degradation and decrease bond strength. ${ }^{17}$ The dissimilar coefficients of the resin-based polymer and the thermal expansion of porcelain are possibly responsible for decreasing the bond strength at the interface of the 2 materials during the alternation series of compression and expansion. ${ }^{17}$

The SBS test was performed in this study, as it enables the assessment of different substrates and has high reliability. On the other hand, anterior teeth are primarily subjected to shear loads and this test enables the comparison of the bond strength values; thus, it was selected for the present study.

Also, despite numerous laboratory studies, there is insufficient information for predicting the clinical performance of the repair systems. Hence, further studies are required to determine the repair bond strength of zirconia. In addition, clinical studies are needed to confirm the results of the in vitro tests.

\section{Conclusions}

Considering the current results, a separate silanization procedure during porcelain surface treatment led to a significant increase in the repair bond strength of porcelain irrespective of the presence or absence of silane in the composition of universal adhesives. Therefore, a separate application of silane along with HF etching and the application of a universal adhesive for porcelain surface treatment can improve the repair bond strength of porcelain.

\section{ORCID iDs}

Azam Valian (1) https://orcid.org/0000-0001-5363-9762

Elham Moravej Salehi (1) https://orcid.org/0000-0003-4184-1423

Mehrangar Mahmoudzadeh (1) https://orcid.org/0000-0001-5952-0148

Neda Kheirkhah Dabagh (1) https://orcid.org/0000-0003-4352-0316

\section{References}

1. Hickel R, Brüshaver K, llie N. Repair of restorations - criteria for decision making and clinical recommendations. Dent Mater. 2013;29(1):28-50. doi:10.1016/j.dental.2012.07.006

2. Hwang MH, Kim HR, Kwon TY. Repairing fractured ceramic veneer with CAD/CAM ceramic blocks: A preliminary tensile bond strength study. Mater Technol. 2019;34(1):43-50. doi:10.1080/10667857.2018.1495881

3. Hakimaneh SMR, Shayegh SS, Ghavami-Lahiji M, Chokr A, Moraditalab A. Effect of silane heat treatment by laser on the bond strength of a repair composite to feldspathic porcelain. J Prosthodont. 2020;29(1):49-55. doi:10.1111/jopr.12762

4. Borgia Botto E, Baró R, Borgia Botto JL. Clinical performance of bonded ceramic inlays/onlays: A 5- to 18-year retrospective longitudinal study. Am J Dent. 2016;29(4):187-192.

5. Loomans B, Özcan M. Intraoral repair of direct and indirect restorations: Procedures and guidelines. Oper Dent. 2016;41(S7):S68-S78. doi:10.2341/15-269-LIT

6. Özcan M. Fracture reasons in ceramic-fused-to-metal restorations.JOral Rehabil. 2003;30(3):265-269. doi:10.1046/j.1365-2842.2003.01038.x

7. Kanzow P, Wiegand A, Schwendicke F, Göstemeyer G. Same, same, but different? A systematic review of protocols for restoration repair. J Dent. 2019;86:1-16. doi:10.1016/j.jdent.2019.05.021

8. Yadav JS, Dabas N, Bhargava A, Malhotra P, Yadav B, Sehgal M. Comparing two intraoral porcelain repair systems for shear bond strength in repaired cohesive and adhesive fractures, for porcelainfused-to-metal restorations: An in vitro study. J Indian Prosthodon Soc. 2019;19(4):362-368. doi:10.4103/jips.jips_120_19

9. Amorim R, Vermudt A, Pereira JR, Pamato $S$. The surface treatment of dental ceramics: An overview. J Res Dent. 2018;6(4):80-85. doi:10.19177/jrd.v6e4201880-85

10. Marfenko S, Özcan M, Attin T, Tauböck TT. Treatment of surface contamination of lithium disilicate ceramic before adhesive luting. Am J Dent. 2020;33(1):33-38.

11. Soares LD, Basso GR, Spazzin AO, Griggs J, Moraes RR. Mechanical reliability of air-abraded and acid-etched bonded feldspar ceramic. Dent Mater. 2016;32(3):433-441. doi:10.1016/j.dental.2015.12.011

12. Ahrari F, Boruziniat A, Mohammadipour HS, Alirezaei M. The effect of fractional $\mathrm{CO}_{2}$ laser irradiation on shear bond strength of resin cement to feldspathic porcelain. J Lasers Med Sci. 2018;9(2):101-106. doi:10.15171/jlms.2018.20

13. Hou $Y$, Shen $R$, Chen $L$, et al. Shear bond strength of different CAD/CAM ceramics: Acid vs Er:YAG laser etching. Photomed Laser Surg. 2018;36(11):614-620. doi:10.1089/pho.2018.4475

14. Suárez-Moya DA, Cruz-González AC, Calvo-Ramírez JN. Interaction of a universal adhesive with different surface treatments with feldespathic ceramics. Saudi Dent J. 2019;31(3):350-354. doi:10.1016/j.sdentj.2019.03.008

15. Matinlinna JP, Lung CYK, Tsoi JKH. Silane adhesion mechanism in dental applications and surface treatments: A review. Dent Mater. 2018;34(1):13-28. doi:10.1016/j.dental.2017.09.002 
16. Nagarkar S, Theis-Mahon N, Perdigão J. Universal dental adhesives: Current status, laboratory testing, and clinical performance. J Biomed Mater Res B Appl Biomater. 2019;107(6):2121-2131. doi:10.1002/jbm.b.34305

17. Sriamporn T, Kraisintu P, See LP, Swasdison S, Klaisiri A, Thamrongananskul N. Effect of different neutralizing agents on feldspathic porcelain etched by hydrofluoric acid. Eur J Dent. 2019;13(1):75-81. doi:10.1055/s-0039-1688535

18. Matinlinna JP, Vallittu PK. Bonding of resin composites to etchable ceramic surfaces - an insight review of the chemical aspects on surface conditioning. J Oral Rehabil. 2007;34(8):622-630. doi:10.1111/j.1365-2842.2005.01569.x

19. Cuevas-Suárez CE, de Oliveira da Rosa WL, Vitti RP, da Silva AF, Piva E. Bonding strength of universal adhesives to indirect substrates: A meta-analysis of in vitro studies. J Prosthodont. 2020;29(4):298-308. doi:10.1111/jopr.13147

20. Zaghloul H, Elkassas DW, Haridy MF. Effect of incorporation of silane in the bonding agent on the repair potential of machinable esthetic blocks. Eur J Dent. 2014;8(1):44-52. doi:10.4103/1305-7456.126240

21. Kim RJY, Woo JS, Lee IB, Yi YA, Hwang JY, Seo DG. Performance of universal adhesives on bonding to leucite-reinforced ceramic. Biomater Res. 2015;19:11. doi:10.1186/s40824-015-0035-1

22. Kalavacharla VK, Lawson NC, Ramp LC, Burgess JO. Influence of etching protocol and silane treatment with a universal adhesive on lithium disilicate bond strength. Oper Dent. 2015;40(4):372-378. doi:10.2341/14-116-L

23. Vasconcelos Moro AF, Ramos AB, Rocha GM, dos Reis Perez C. Effect of prior silane application on the bond strength of a universal adhesive to a lithium disilicate ceramic. J Prosthet Dent. 2017;118(5):666-671. doi:10.1016/j.prosdent.2016.12.021

24. AlRabiah M, Labban N, Levon JA, et al. Bond strength and durability of universal adhesive agents with lithium disilicate ceramics: A shear bond strength study. J Adhes Sci Technol. 2018;32(6):580-589. doi:10.1080/01694243.2017.1372932

25. Yao C, Yang H, Yu J, Zhang L, Zhu Y, Huang C. High bond durability of universal adhesives on glass ceramics facilitated by silane pretreatment. Oper Dent. 2018;43(6):602-612. doi:10.2341/17-227-L

26. Altunsoy M, Botsali MS, Korkut E, Kucukyilmaz E, Sener Y. Effect of different surface treatments on the shear and microtensile bond strength of resin-modified glass ionomer cement to dentin. Acta Odontol Scand. 2014;72(8):874-879. doi:10.3109/00016357.2014.919664

27. Akyıl MŞ, Yılmaz A, Bayındır F, Duymuş ZY. Microtensile bond strength of resin cement to a feldspathic ceramic. Photomed Laser Surg. 2011;29(3):197-203. doi:10.1089/pho.2009.2746

28. Shirinzad M, Fekrazad R, Rezaei-Soufi L, Yarmohammadi E, Jalalian S. Evaluation of shear bond strength of composite to feldspathic porcelain after porcelain surface treatment with $\mathrm{CO}_{2}$ and Er:Yag lasers. J Islam Dent Assoc Iran. 2013;25(3):134-138.

29. Yoshida Y, Yoshihara K, Nagaoka N, et al. Self-assembled nanolayering at the adhesive interface. J Dent Res. 2012;91(4):376-381. doi:10.1177/0022034512437375

30. de Melo RM, Valandro LF, Bottino MA. Microtensile bond strength of a repair composite to leucite-reinforced feldspathic ceramic. Brazil Dent J. 2007;18(4):314-319. doi:10.1590/s0103-64402007000400008 